\title{
Problems of Obesity in Drivers and Road Safety
}

\author{
IV Fedotova* \\ Holder of Habilitation degree in Medicine, Head of the Hygiene Department of FBSI, Russia
}

Received: 畊 January 11, 2019; Published: 盋January 23, 2019

*Corresponding author: Fedotova Irina Viktorovna, Holder of Habilitation degree in Medicine, head of the Hygiene Department of FBSI, Russia

\section{Opinion}

At the present time obesity is one of the most important medical, social and economic problems of contemporary society, which lead to a reduction in life expectancy and degeneration of its quality. It is called "non-communicable epidemic of the 21st century". Over the past 20-25 years, both in developed and developing countries, the number of people suffering from not merely overweight, but obesity, has significantly increased. Abdominal obesity is one of the major indicators in the diagnosis of metabolic syndrome (MS), which is regarded to be a risk factor for the development of such severe pathologies as coronary heart disease and diabetes. Numerous studies have also shown the negative impact of obesity on labour productivity; its increasing prevalence requires an increase in financial expenditures on health care.

Drivers of motor transport are the most numerous and stable professional group among men of working age. The state of health determines their performance efficiency and, therefore, road safety. Driving a vehicle is accompanied by a high level of neuroemotional stress, which is the leading factor in this professional activity, provoking and supporting chronic stress. Against the background of reduced physical activity and malnutrition, stress is considered as the main cause of a significant proportion of drivers with pre-obesity with a tendency to put on weight further. This fact, according to some authors, leads to the need to make changes in the construction of the car cabin, taking into account the increased anthropometric measurement of drivers. It was also determined that severe pathologies of the cardiovascular and endocrine systems which are frequently registered in this occupational group can cause rapid deterioration in the health condition of drivers who are at work and result in accidents on the road.

In the aspect of this problem, the data obtained by the authors in the study of 133 truck drivers engaged in the transportation of trucks in the city of Nizhny Novgorod (Russia) is of interest. It should be noted that a significant proportion of them are over 50 years old $(64.2 \%)$ and with work experience of 30 years or more (67.1\%); the average age of drivers was $50.76 \pm 0.72$ years, the driving experience $-30.73 \pm 0.76$ years. Overweight and obesity were determined by calculating body mass index. Analysis of the prevalence of MS was conducted in the following age groups: up to 40 years (22 people), 40-49 (40), 50-59 (82), and 60 years or more (29). According to the occupational experience, the drivers were also divided into 4 groups: 1 st - with work experience less than 20 years (28 people), 2nd - 20-29 (29), 3rd - 30-39 (92), 4 th - over 40 years old (24). For the diagnosis of MS the following criteria were used: the working criteria of the Committee of Experts of the US National Cholesterol Education Program on the detection, evaluation and treatment of high blood cholesterol in adults (Adult Treatment Panel III, ATP III), which are the most adapted to clinical practice. According to the ATP III classification, MS is diagnosed when there are abnormalities in any of the three or more of the five symptoms: abdominal obesity (waist circumference), triglycerides, high-density lipoprotein cholesterol, arterial pressure (AP), fasting plasma glucose. Working conditions in the studied group of truck drivers were characterized by exposure to the levels of noise and vibration increased relatively to the normative microclimate.

When driving on major highways during the period of intensive traffic, concentrations of aliphatic hydrocarbons, carbon monoxide and solid particles exceeding the maximum permissible levels were recorded in the cabs. According to the analysis of the conducted questionnaire, an unbalanced diet was established in this group (unreasonable day regimen, excessive consumption of fats, alcohol) and low physical activity - only $6.6 \%$ of truck drivers were engaged in fitness (swimming, running, morning gymnastics). Among the examined drivers, $33.5 \pm 3.6 \%$ had a normal weight, the proportion of overweight persons was $66.4 \pm 3.6 \%$, including those with overweight $-38.7 \pm 3.7 \%$, and with obesity of varying degrees $27.7 \pm 3.4 \%$. It should be noted that the proportion of obese people increased with age and was respectively $13.6 \pm 7.7 ; 22.5 \pm 6.6 ; 34.1$ 
\pm 5.2 and $27.7 \pm 3.4$, and the differences are significant between the first and third groups t1-3 $=2.2(\mathrm{p}<0.05)$.

During the examination, metabolic syndrome, i.e. the presence of abdominal obesity and two or more markers mentioned above was diagnosed in 65 drivers (37.6 $\pm 3.7 \%$ ), which was almost 2 times higher than the average statistical indicators. The most frequent symptom of MS in the group of drivers was the combination of abdominal obesity with hypertension. With increasing age, there was a gradual growth of the frequency of MS, reaching 1.8 times ( $p>0.05)$ in the oldest age group compared with the youngest. When analyzing the frequency of MS according to experience, a sharp increase was noted in the 4 th group relative

ISSN: 2574-1241

DOI: 10.26717/BJSTR.2019.13.002409

Fedotova Irina Viktorovna. Biomed J Sci \& Tech Res

This work is licensed under Creative Commons Attribution 4.0 License

Submission Link: https://biomedres.us/submit-manuscript.php to the first three (2.5, 2.3 and 1.6 times, respectively) and these differences are statistically significant. Thus, this study showed that the peculiarities of the occupational activity of truck drivers increase the risk of metabolic syndrome development. Taking into account the drastic consequences of MS presence on health, it is necessary to take active preventive measures aimed at reducing the risk of developing MS in drivers, for at the early stage of metabolic disorders it is possible to prevent the transition of this symptom complex to more severe life-threatening pathological conditions. The system of prevention should include such areas as a reduction of the stress factors influence, the introduction of educational health programs, including the basics of a healthy lifestyle, sensible nutrition, and high physical activity.

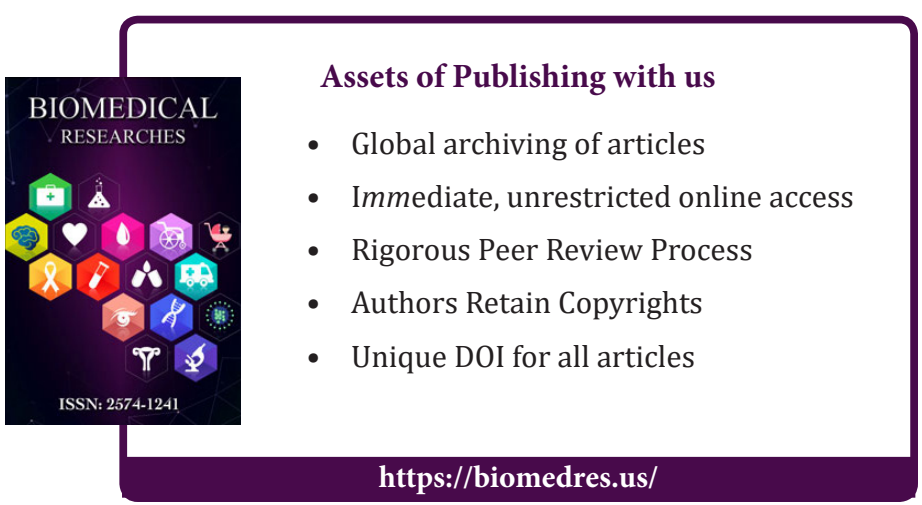

